Peer Reviewed Paper openaccess

\title{
Innovative technique for analysis of wastewater contaminants using hyperspectral imaging
}

\author{
Ramy Abdlaty, ${ }^{\mathrm{a}, *}$ Mohamed Gobara, ${ }^{\mathrm{b}}$ Ibrahim Naiem ${ }^{\mathrm{b}}$ and Mohamed Mokhtar ${ }^{\mathrm{b}}$ \\ aBiomedical Engineering, Military Technical College, Cairo, Egypt \\ ${ }^{\mathrm{b} C h e m i c a l ~ E n g i n e e r i n g, ~ M i l i t a r y ~ T e c h n i c a l ~ C o l l e g e, ~ C a i r o, ~ E g y p t ~}$ \\ Contacts \\ Ramy Abdlaty: ramy.elghwas@mtc.edu.eg, \\ https://orcid.org/0000-0002-8933-1724 \\ Mohamed Mokhtar: m.mokhtar@mtc.edu.eg \\ Ibrahim Naiem: Ibrahim.naiem@mtc.edu.eg \\ Mohamed Gobara: m.gobara@mtc.edu.eg, \\ https://orcid.org/0000-0001-5283-2165
}

\begin{abstract}
Accurate, fast, quantitative on-site analysis of wastewater is an environmental necessity. Therefore, we report a quantitative spectroscopic study in order to analyse organic contaminants in wastewater. For this purpose, two spectroscopy-based techniques were utilised and their results are compared. The first technique is ultraviolet/visible (UV/vis) spectrophotometry. This technique is a standard laboratory technique, however, it is inappropriate for working in the field. Besides, the analysis results of the standard laboratory technique display low accuracy in detecting small concentrations of wastewater contaminants such as methylene blue ( $20 \mathrm{ppm})$. Accordingly, there is a need for another technique to overcome the shortcomings of UV/vis spectrophotometry. Hyperspectral imaging (HSI) combines imaging and spectrometry. HSI works on-site since it is portable and easy to operate. The analysis of the data acquired by HSI showed a higher efficacy in accurately detecting both low ( $20 \mathrm{ppm})$ and high concentrations (>50 ppm) of water contaminants such as methylene blue and methyl orange. For verification, Pearson correlation coefficients were computed between the concentration of contaminants and their absorbance for all the operating spectral bands. The results of the two techniques were statistically compared using Bland and Altman's limit of agreement. This study showed that HSI is potentially a promising technique for the analysis of wastewater contaminants on-site.
\end{abstract}

Keywords: spectrophotometry, hyperspectral imaging, chemical dyes, methylene blue, methyl orange

\section{Introduction}

The past half-century has witnessed a significant increase in the disposal of industry-produced wastewater into the Earth's natural water resources due to the immense growth of industrial establishments. Industrial wastewater contains a variety of contaminants which can be solids, liquids, inorganic and organic materials. ${ }^{1}$ Among these contaminants, organic materials require particular care when determining the level of water contamination on-site. ${ }^{1}$ Organic pollutants, such as manufacturing dyes including methylene blue (MB) and methyl orange
Correspondence

R. Abdlaty (ramy.elghwas@mtc.edu.eg)

Received: 7 August 2020

Revised: 1 October 2020

Accepted: 9 October 2020

Publication: 21 October 2020

doi: 10.1255/jsi.2020.a12

ISSN: $2040-4565$

\begin{abstract}
Citation
R. Abdlaty, M. Gobara, I. Naiem and M. Mokhtar, "Innovative technique for analysis of wastewater contaminants using hyperspectral imaging", J. Spectral Imaging 9, a12 (2020). https://doi.org/10.1255/jsi.2020.a12

(C) 2020 The Authors

This licence permits you to use, share, copy and redistribute the paper in any medium or any format provided that a full citation to the original paper in this journal is given, the use is not for commercial purposes and the paper is not changed in any way.
\end{abstract}


(MO), are among the most common organic contaminants in wastewater. These dyes are commonly used in the production of many goods such as leather, paper, detergents and cosmetics. ${ }^{2}$ If untreated, organic contaminants, which flow to water sources with the effluent stream, pose a serious hazard to all living creatures when they reach a certain concentration: the discharge of large amounts of chemical dyes in industrial effluent can lead to a highly toxic effect. ${ }^{3}$ Organic contaminantion also requires expensive treatment of water due to problems such as high turbidity, bad odour and adverse colour.

A high concentration of organic contaminants in natural water decreases the penetration of sunlight, which is required for photosynthesis by some aquatic microorganisms. ${ }^{4}$ Moreover, dye contaminants decrease oxygen dissolution in water by preventing gaseous oxygen from crossing the air-water interface. Rather, they exert an oxygen demand. Hence, marine life can be affected harshly due to dissolved oxygen depletion. ${ }^{4}$ If the contaminated water reaches soil, it will clog its pores and the roots of the existing plants will cease to grow. ${ }^{5}$ Furthermore, $\mathrm{MB}$ and $\mathrm{MO}$ are carcinogenic if they reach the human body through drinking water, absorption through the skin or the ingestion of contaminated food. ${ }^{6}$ Thus, MB and MO were selected in this study as organic dyes heavily produced in large amounts by industry. ${ }^{7}$ Industries such as fabric, medicinal, leather, foodstuff production, cosmetic and paint release into the environment approximately $10-15 \%$ of the more than 800,000 tons of distinct types of dyes manufactured worldwide each year. ${ }^{8}$

MBis a cationic dye distinguished by a remarkable change in its optical properties when aggregated in a sample. ${ }^{9}$ For instance, it exhibits two absorption peaks around $293 \mathrm{~nm}$ and $664 \mathrm{~nm}$ in diluted aquatic solutions. ${ }^{10}$ The chemical structure of $\mathrm{MB}$ is responsible for its absorption peaks. To explain more, the MB monomer displays an absorption peak around $664 \mathrm{~nm}$, for the MB dimer structure this peak moves to $605 \mathrm{~nm}$ and in the MB trimer the peak moves to $575 \mathrm{~nm} .{ }^{10}$ The molar coefficients of absorption for all $\mathrm{MB}$ chemical structures were reported to be in the range from 40 to $90 \times 10^{3} \mathrm{M}^{-1} \mathrm{~cm}^{-1} \cdot{ }^{10} \mathrm{MO}$ dyes belong to the azo group of anionic dyes. ${ }^{11}$ This group includes the nitrogen chromophore and the aromatic structure, as such it is cumbersome to extract it from wastewater either by chemical or biological degradation. ${ }^{11}$

International environmental and health organisations have established guidelines limiting the concentration of contaminants in wastewater produced by industry. ${ }^{12}$ For this purpose, monitoring the concentrations of the industrial effluent is essential. Different methods are used to accomplish this goal by analysing samples either on-site or at in-field laboratories. A popular approach for precise qualitative and quantitative analysis of the chemical dyes in aqueous solutions is ultraviolet/visible (UV/vis) spectroscopy. ${ }^{2,10,13}$ The UV/vis spectrum has sufficient energy to excite valence electrons in various atoms and molecules. Spectrophotometers are highly reliable and precise in spectral measurements, as such they are considered the gold standard for many chemical analysis procedures. However, spectrophotometers are not flawless. For instance, they are less favoured for use outdoors due to being bulky, requiring time-consuming sample preparation and being highly sensitive to the surrounding optical and electronic noise. ${ }^{14}$

Compared to spectrophotometry, hyperspectral imaging (HSI) has a lower spectral resolution. However, $\mathrm{HSI}$ is very consistent in spectral measurements in addition to being entirely suitable for outdoor measurements, simple to maintain and easy to use. The analysis of HSI data is typically customised for each case so as to achieve classification of the particular objects of interest to the user. ${ }^{15-17} \mathrm{HSI}$ combines imaging and spectroscopy in one platform. This combination produces a three-dimensional data format. ${ }^{18,19}$ This data includes two-dimensional spatial information along a third spectral dimension to form a datacube. The datacube's spatial information is contained in an image of the object, and its spectral information is included in capturing the same image using a sequence of spectral bands. A great advantage of HSI is providing a spectral identity for every single pixel enclosed in the field of view. For that advantage, HSI finds application in divergent fields of research such as in agriculture, ${ }^{20,21}$ nutrients production, ${ }^{22,23}$ space science, ${ }^{15}$ forensic medicine ${ }^{24}$ and health science. ${ }^{25-28}$ To the best of our knowledge, there has been no report in the literature of the use of HSI for quantitative chemical analysis on-site. The present work is a steady accumulation of the ongoing research regarding the application of $\mathrm{HSI}$ in the chemical analysis field of research. In this work, HSI is employed to quantitatively analyse two wastewater organic contaminants, $\mathrm{MB}$ and $\mathrm{MO}$, since they are produced in large quantities by factories. This work reports a comparative study between HSI and UV/vis spectrophotometry for monitoring wastewater 
contamination. For the comparison, various aqueous concentrations of the two contaminants were prepared, measured, imaged and finally quantitatively analysed. Figure 1 displays a schematic diagram for the two techniques compared in this work.

\section{Materials and methods}

\section{Preparation of dye samples}

The organic dyes used in this study, $\mathrm{MB}$ and $\mathrm{MO}$, were purchased from Sigma-Aldrich (St Louis, Missouri, USA). ${ }^{9}$ The $\mathrm{MB}$ and $\mathrm{MO}$ dyes did not undergo any purification process after being purchased. The water used for sample preparation was distilled using the reverse osmosis procedure following normal laboratory procedures. ${ }^{10}$ The aqueous dye solutions were meticulously prepared from recently purchased $\mathrm{MB}$ and $\mathrm{MO}$ stocks. The dilution of the dye solution was carried out at room temperature $\left(24 \pm 1^{\circ} \mathrm{C}\right)$. The $\mathrm{pH}$ value was measured to be $7.48 \pm 0.13$ for $\mathrm{MB}$ samples and $7.95 \pm 0.19$ for $\mathrm{MO}$.

$\mathrm{MB}$ and $\mathrm{MO}$ samples were prepared in the laboratory in an ample quantity $(0.25 \mathrm{~L})$ for each concentration listed in Table 1. The prepared quantity is calculated to provide three samples $\left(2 \mathrm{~cm}^{3}\right)$ for the UV/vis spectrophotometer, three other samples $\left(10 \mathrm{~cm}^{3}\right)$ for the HSI system and a sufficient amount for any accidental loss. The process of preparing this quantity $(0.25 \mathrm{~L})$ for each dye concentration was repeated two times over the elapsed time of the study. The temperature and $\mathrm{pH}$ level for the dye samples were maintained in order to prevent $\mathrm{MB}$ and MO dimerisation.?

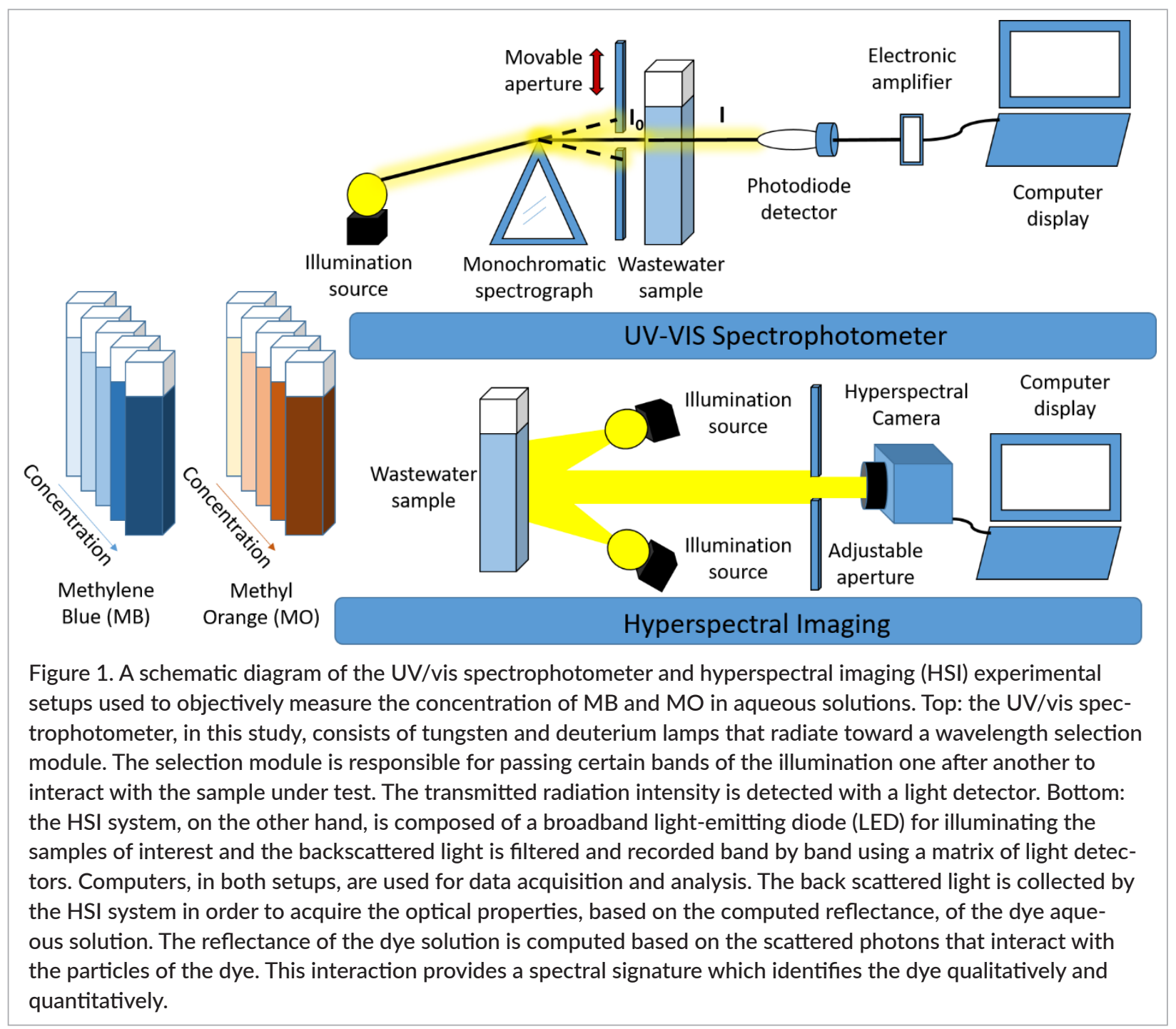


Table 1. The set of the prepared cationic and anionic dye aqueous solutions

\begin{tabular}{|c|c|c|}
\hline $\begin{array}{c}\text { Dye aqueous } \\
\text { solution }\end{array}$ & Methylene blue & Methyl orange \\
\hline 1 & $10 \mathrm{ppm}$ & $10 \mathrm{ppm}$ \\
\hline 2 & $12 \mathrm{ppm}$ & $12 \mathrm{ppm}$ \\
\hline 3 & $14 \mathrm{ppm}$ & $14 \mathrm{ppm}$ \\
\hline 4 & $16 \mathrm{ppm}$ & $16 \mathrm{ppm}$ \\
\hline 5 & $18 \mathrm{ppm}$ & $18 \mathrm{ppm}$ \\
\hline 6 & $20 \mathrm{ppm}$ & $20 \mathrm{ppm}$ \\
\hline 7 & $30 \mathrm{ppm}$ & $30 \mathrm{ppm}$ \\
\hline 8 & $40 \mathrm{ppm}$ & $40 \mathrm{ppm}$ \\
\hline 9 & $50 \mathrm{ppm}$ & $50 \mathrm{ppm}$ \\
\hline
\end{tabular}

\section{Absorption spectrophotometer}

The study measurements were carried out in one day for all the samples of each dye group to preserve the same conditions (temperature and humidity). All the samples, including distilled water samples, were poured into quartz cuvettes, of $1 \mathrm{~cm}$ thickness, immediately before the absorption spectra measurements. The absorption spectra of the aqueous $\mathrm{MB}$ and $\mathrm{MO}$ samples were measured three times consecutively on the spectrophotometer (UV-1800, TOMOS, USA) according to the standard operating procedure.

\section{Hyperspectral imaging system}

Typically, an HSI optical setup employs a broadband illumination source emitting radiation in the spectral region of interest. The illumination source employed for this study was a light-emitting diode (LED) white lamp (wavelength range $400-700 \mathrm{~nm}$ ). The reason for the employment of the LED source is our interest in the visible region of the optical spectrum. The HSI-collected data was, in this study, captured via a portable SOC-710 hyperspectral imaging camera (Surface Optics Corporation, San Diego, CA, USA). The SOC-710 features a 12-bit image sensor (high signal-to-noise ratio charge-coupled device) with an average spectral resolution of $\sim 5.3 \mathrm{~nm}$. The sensor operates in ambient/external lighting conditions using variable times of exposure and electronic gain. The SOC-710 camera is capable of acquiring spectral data in the range of $380-1051 \mathrm{~nm}$ in 128 consecutive bands.

$\mathrm{HSI}$ data consist of an uninterrupted sequence of images for the field of view (FOV), yet each image is taken at a distinct spectral band. The spectral sequence of images for the FOV is termed a datacube, since it is a three-dimensional matrix. The hyperspectral datacube, produced by the SOC-710 model of hyperspectral imaging camera, has the following dimensions: $x=696$ pixels, $y=520$ pixels and $z=128$ spectral bands). The spectral profile computed for each dye sample is equal to the average reflectance of seven pixels selected along the test tube we used to hold the sample from the top to the bottom. The seven pixels are separated by 30 pixels along the longitudinal axis of the test tube.

Two datacubes, other than the object's one, are captured as a rule: one for the dark current of the image sensor and the second for a white standard diffuse reflectance surface. Using the three datacubes, the reflectance for the object of interest is computed. Initially, the dark current pixel intensities $\left(I_{d c}\right)$ are subtracted from both the corresponding object $\left(I_{o b j}\right)$ and the white surface $\left(I_{w s}\right)$ pixel intensities. Afterwards, the noise-free object's datacube is compensated for uneven illumination by dividing it by the corresponding pixel intensities for the white standard. Accurate compensation necessitates preserving the exact environment of imaging for all the datacubes. Summing up, the object reflectance $\left(R_{o b j}\right)$ is calculated as shown in Equation 1). The selection of the user-defined pixels and the computed-based-reflectance were done with custom code written in MATLAB (The Mathworks Inc., Natick, Massachusetts, USA).

$$
R_{o b j}=\frac{I_{o b j}-I_{d c}}{I_{w s}-I_{d c}}
$$

\section{Experimental work and results Absorption experiments}

The experiments were carried out on two groups of aqueous solutions, one for $\mathrm{MB}$ and the other for $\mathrm{MO}$ dye. The experimental work was performed at room temperature and ambient humidity. The $\mathrm{pH}$ of the aqueous solutions was standardised by taking the average of multiple points on the calibration curve measurements. The absorbance value was computed according to the BeerLambert law:

$$
A=\varepsilon b C
$$

where $A$ is the absorbance, $\varepsilon$ is the molar absorptivity $\left(\mathrm{M}^{-1} \mathrm{~cm}^{-1}\right), b$ is the width of the cuvette carrying the sample and $C$ is the instantaneous concentration of the dye in the solution. Using Equation (2), the spectral profiles 

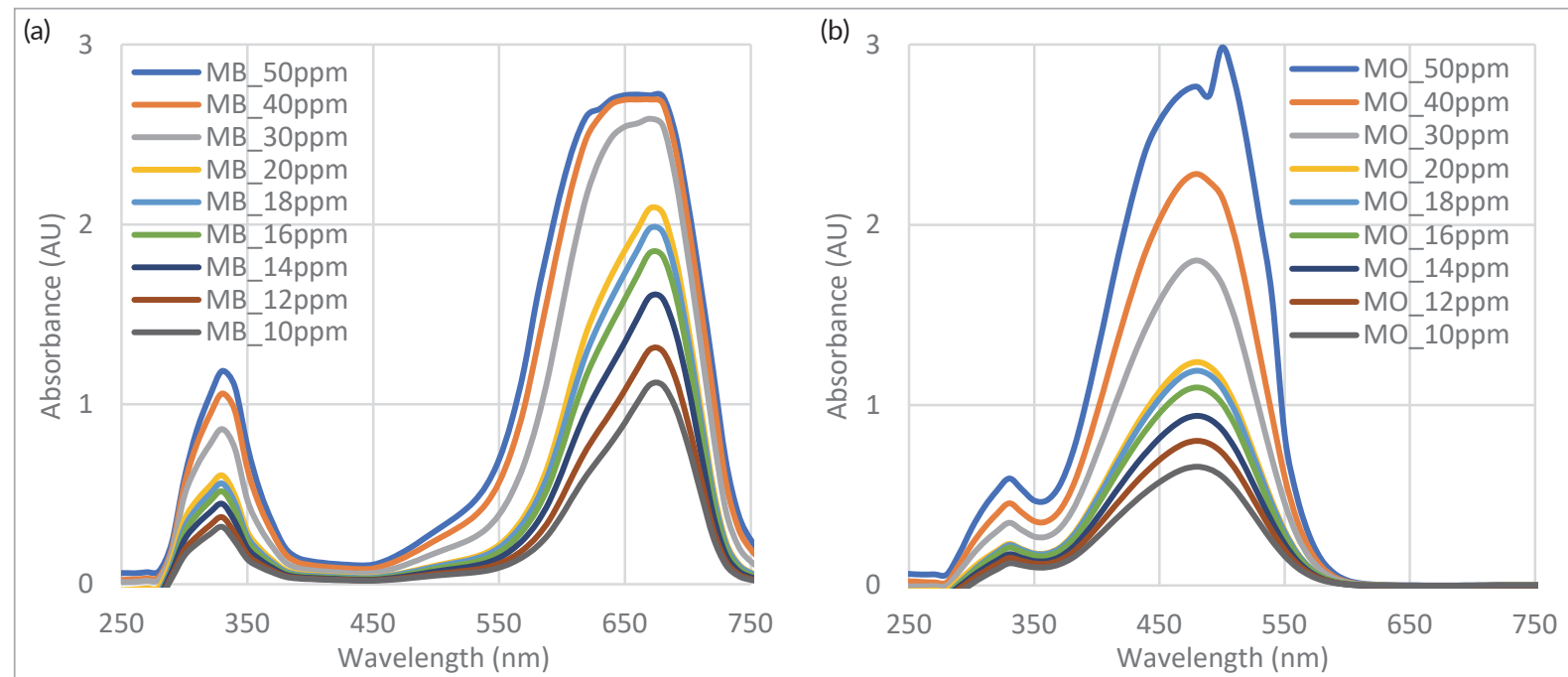

Figure 2. The measured absorption spectra for (a) MB samples (10, 12, 14, 16, 18, 20, 30, 40 and 50 ppm), (b) MO samples $(10,12,14,16,18,20,30,40$ and $50 \mathrm{ppm})$ using the UV/vis spectrophotometer.

for the samples of both $\mathrm{MB}$ and $\mathrm{MO}$ in the concentration range of $10-50 \mathrm{ppm}$ were obtained from the UV/vis spectrophotometer measurements. The spectral profiles for both the $\mathrm{MB}$ and the $\mathrm{MO}$ dyes are displayed in Figure 2(a, b), respectively.

The UV/vis spectrophotometer measurements for the $\mathrm{MB}$ and $\mathrm{MO}$ dyes exhibited a higher absorption at the wavelengths of $660 \mathrm{~nm}$ and $440 \mathrm{~nm}$, respectively. Figure 3 displays the relation between the computed absorbance and the concentration for each dye solution at its distinguished absorption spectral band. According to Equation (2) this relation should be linear. However, the absorption measurements for the MB samples showed an obvious non-linearity in the absorption/concentration relationship. This non-linearity pointed to the low accuracy of the UV/vis spectrophotometer in detecting the high concentrations of some dyes, including $\mathrm{MB}$ in aqueous solutions as shown in Figure 3(a).The observed non-linearity of the UV/vis spectrophotometer also accounts for the poor performance of the device for dilute dye solutions.
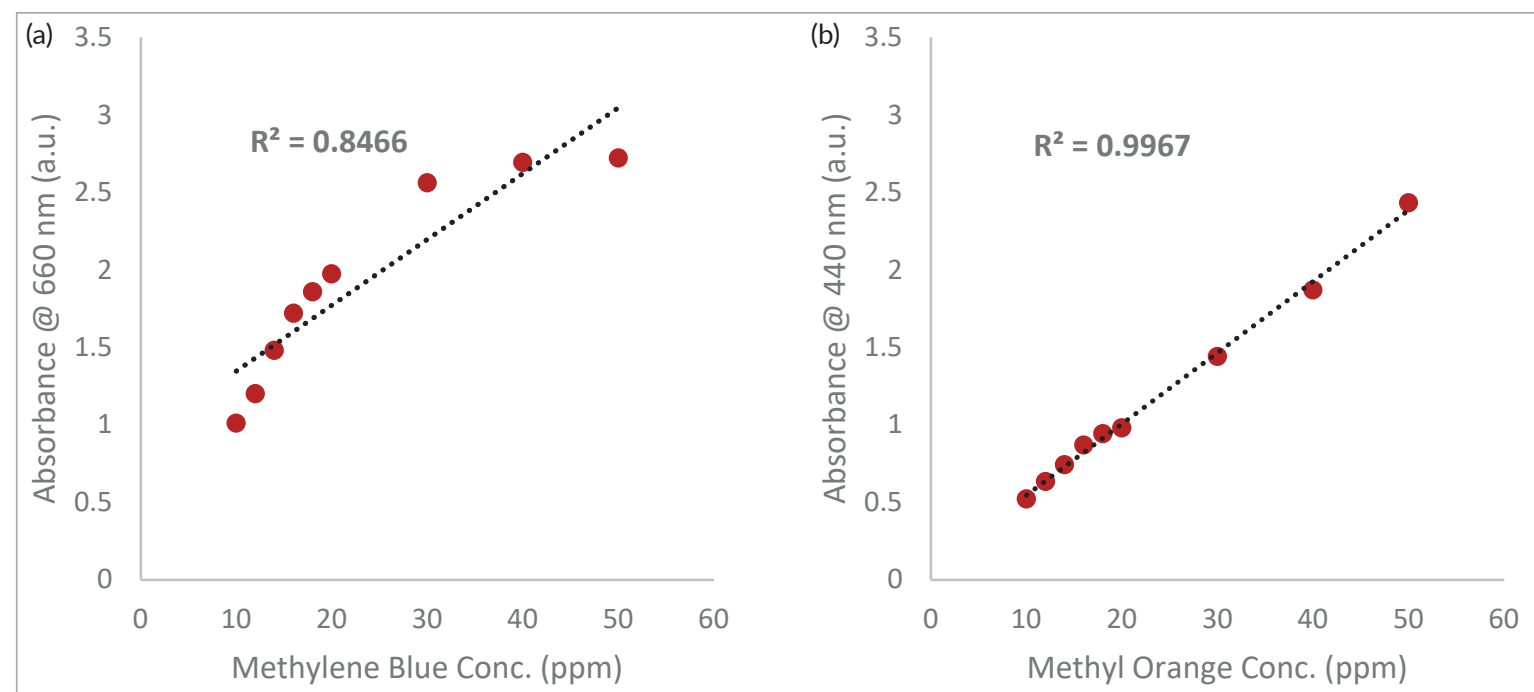

Figure 3. (a) the absorbance measured at $660 \mathrm{~nm}$, using the UV/vis spectrophotometer for the group of MB aqueous solutions with concentrations of $10,12,14,16,18,20,30,40$ and 50 ppm. (b) the absorbance measured at $440 \mathrm{~nm}$ for the group of $\mathrm{MO}$ aqueous solutions with concentrations of $10,12,14,16,18$, 20, 30, 40 and 50 ppm. Both graphs have linear fitting in black dots to estimate how close the absorbance measurements of the dye samples are related to its concentrations. 


\section{Hyperspectral imaging and data processing}

All the dye samples prepared for this study were imaged and their reflectance was computed three times. The reason for repeating the computation is the minimisation of possible random errors. Based on the reflectance, the absorbance is calculated using Equation (3), where $A_{o b j}$ signifies the absorbance of the dye sample using HSI-collected data.

$$
A_{o b j}=\log \left(\frac{1}{R_{o b j}}\right)
$$

Figure 4 displays the average computed absorbance for the MB and MO samples listed in Table 1.

Following the absorbance computation, it is of interest to identify the distinguishing spectral band that highlights the change of the dye concentration and verify that with the spectrophotometer. For this, Pearson correlation coefficients were calculated for all the spectral bands captured by the HSI camera against the concentrations of the dyes in all samples. The spectral band that had the highest correlation coefficients was the optimal one. The absorbance of the dye samples in the optimal band was considered the reference for comparison between HSI and the spectrophotometer as shown in Figure 5.

\section{Discussion}

The results of the absorption experiment demonstrated that UV/vis spectrophotometry is limited in the precise identification of the different dye aqueous solutions as shown in Figure 3(a). Beer-Lambert's law implies a linear relationship, however, this relation is contravened for molecules of large size and heavy molecular weight including certain chemical dyes. This contravention is attributed to the interaction between analyte molecules. These interactions of the dye molecules are controlled by intermolecular attractive forces such as hydrogen bonding and van der Waals forces. Such forces change the absorptivity of the analyte and result in a non-linear response as concentration increases. Added to the former, changes in the solution refractive index ( $n$ ) with analyte concentration probably alters the position, size and solid angle of the captured image upon the detector.

The UV/vis spectrophotometer performance in differentiating the MB samples in the concentration range of $10-50 \mathrm{ppm}$ is less accurate compared to the lower range of $10-20$ ppm, as shown in Figure 5(a). So, we tried to find an alternative method that could differentiate accurately between the high and low concentrations of dye-based MB samples. HSI should be useful for this since it is a combination of spectrophotometry and imaging in one platform. Thus, it
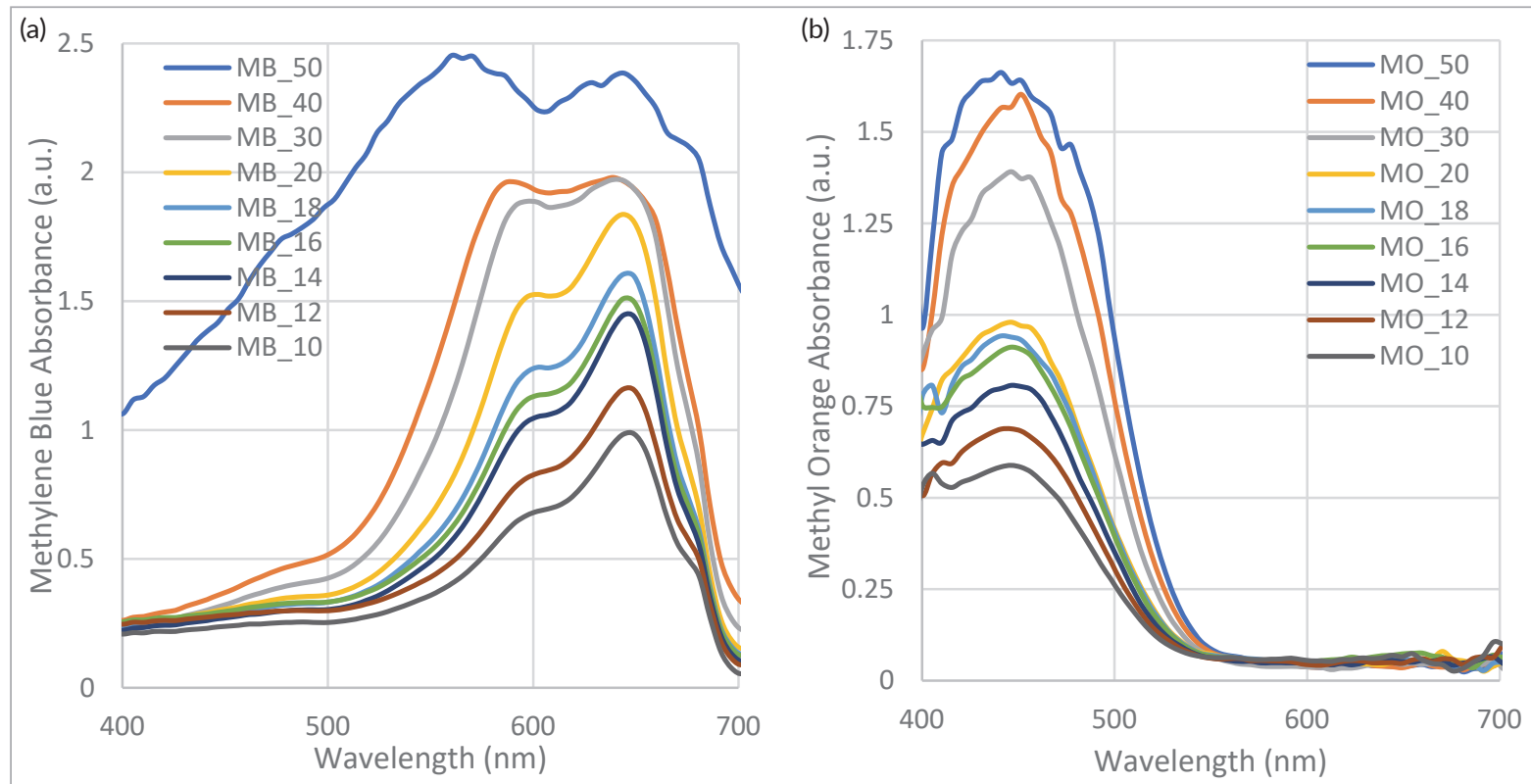

Figure 4. Results of the hyperspectral imaging of both $M B$ and $M O$ aqueous solutions. (a) the computed average absorbance for the group of $\mathrm{MB}$ with concentrations in the range of $10-50 \mathrm{ppm}$ along the spectral range of $350-700 \mathrm{~nm}$ and (b) the computed average absorbance for the group of $\mathrm{MO}$ with concentrations in the range of $10-50 \mathrm{ppm}$ along the spectral range of $400-600 \mathrm{~nm}$. 

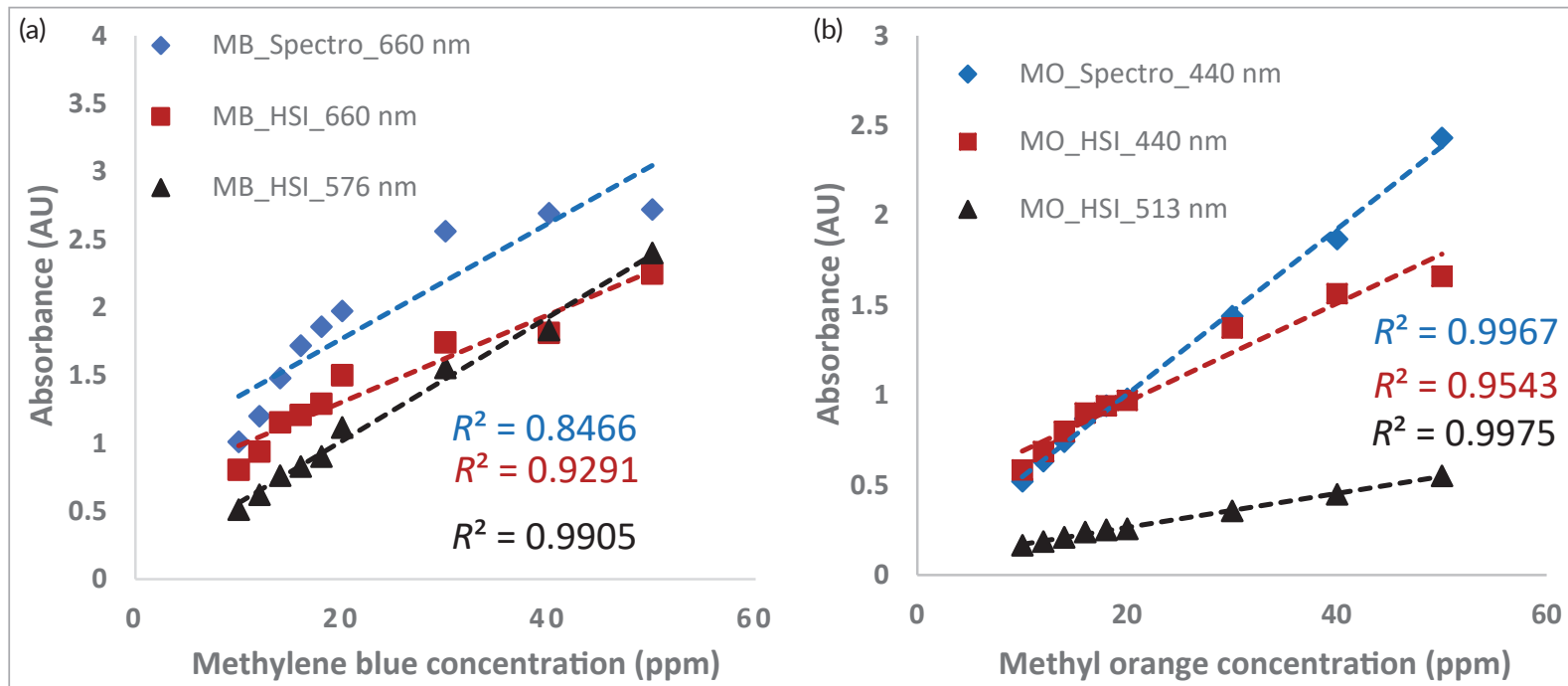

Figure 5. (a) the maximum absorbance, detected at $660 \mathrm{~nm}$, of MB using the UV-VIS-1800 spectrophotometer combined with linear fitting (blue dashed line), and the corresponding absorbance of the SOC-710 HSI camera, captured at $660 \mathrm{~nm}$ and $571 \mathrm{~nm}$ bands combined with linear fitting (red and black dashed lines, respectively). (b) the maximum absorbance, detected at $440 \mathrm{~nm}$, of MO using the UV-VIS-1800 spectrophotometer combined with linear fitting (blue dotted line), and the corresponding absorbance of the SOC-710 HSI camera, captured at $440 \mathrm{~nm}$ and $513 \mathrm{~nm}$ bands combined with linear fitting (red and black dashed lines, respectively). The $R^{2}$ value (coefficient of determination) is displayed for each group of data points.

simultaneously provides spectral and spatial information. Using this information, HSI is able to quantitatively differentiate between the different solutions with variable concentrations. For verification purposes, a comparison between the computed absorbance of the dye-based solutions, using spectrophotometry and $\mathrm{HSI}$, is shown in Figure 5(a and b, respectively). The computed absorbance, in Figure 5, shows a very close performance of HSI compared to the spectrophotometer, in differentiating between the MB samples (10-20 ppm) and the MO samples (10-50 ppm). However, HSI out-performs the spectrophotometer in differentiating the high concentrations of MB samples (20-50 ppm), as shown in Figure 5(a). The spectrophotometer identified the wavelengths $660 \mathrm{~nm}$ and $440 \mathrm{~nm}$ for $\mathrm{MB}$ and $\mathrm{MO}$ maximum absorbance, respectively. However, these bands were not the ones with the highest correlation with $\mathrm{MB}$ and $\mathrm{MO}$ dyes using the HSI system. HSI was able to better distinguish the different concentrations of $\mathrm{MB}$ and $\mathrm{MO}$ at bands close to the one identified by the spectrophotometer as shown in Figure 5( $\mathrm{a}$ and b) at $576 \mathrm{~nm}$ and $513 \mathrm{~nm}$, respectively.

Beside the experimental analysis, it was informative to measure the degree of agreement of HSI compared to UVI vis spectrophotometry in order to assess HSI reliability.
For this purpose, a correlation between the computed absorbance of one group of dye-based samples, based on UV/vis spectrophotometry and HSI, was computed. The MO group of samples was used for computing the correlation coefficients, since the computed absorbance from both spectroscopic techniques is similar. The correlation coefficients were plotted against a diagonal line, representing the state of equality between the two instruments, with angle of $45^{\circ}$, as shown in Figure 6. The computed correlation coefficient indicated a very close agreement for the measured $\mathrm{MO}$ samples using the two methods investigated in this study. Thus, HSI is likely to be a reliable alternative technique to UV/vis spectrophotometry for both qualitative and quantitative in situ analysis.

Bland and Altman's limits of agreement (LOA) is a reliable statistical tool used to check the agreement between two techniques used for the same purpose. The obtained results from $\mathrm{HSI}$ and UV/vis spectrophotometry were the input data for the Bland and Altman's LOA in this study. The LOA are depicted in Figure 7. Since the results from $\mathrm{HSI}$ and UV/vis spectrophotometry lie between the upper and lower LOA, HSI is statistically verified as a suitable technique to replace UV/vis spectrophotometry for in situ analysis of dye samples. 


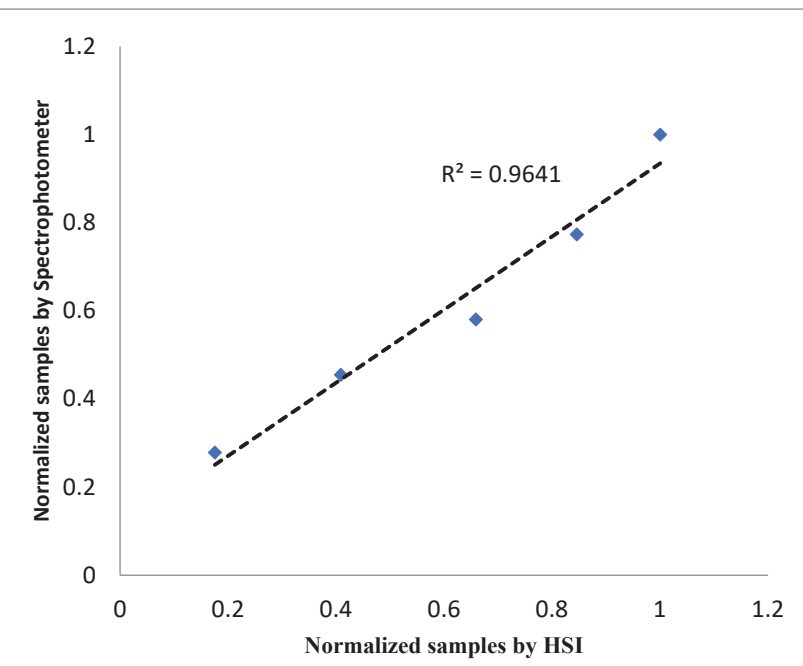

Figure 6. The correlation between the normalised measurements by UV/vis spectrophotometry and the corresponding ones by HSI for the MO group of samples.

\section{Conclusion}

This study confirms the consistency of using HSI as a quantitative analysis technique to detect and quantify organic wastewater contaminants. Dye samples of both $\mathrm{MB}$ and $\mathrm{MO}$ underwent analysis by UV/Vis spectrophotometry and $\mathrm{HSI}$, which were shown to be precise, simple, rapid and low-cost methods for analysing samples for wastewater organic contaminants in the laboratory. In fact, UV/vis spectrophotometry is one of the standard methods used for analysing contaminated water. However, by experiment, it is found that the spectrophotometer has certain limitations. These limitations were clear while evaluating the presence of industrial dyes like MB in water. We hypothesised that hyperspectral imaging could overcome the limitations of UV/Vis spectrophotometry in detecting dyes present in water like $\mathrm{MB}$ and $\mathrm{MO}$.

For verification purposes, nine distinct concentrations of both $\mathrm{MB}$ and $\mathrm{MO}$ dyes in aqueous solutions were accurately prepared in the chemistry laboratory using standard laboratory procedures. The prepared dye samples were split into two groups, one tested by UV/vis spectrophotometry and the other by HSI. We concluded:

1. UV/vis spectrophotometry is a standard technique for analytical chemistry, but it is limited in detecting low concentrations of dyes like MB in aqueous solutions.

2. UV/vis spectrophotometry is limited to small amounts of samples that fit into the standard cuvette of the device. For large amounts or numbers of samples, experiments need to be repeated several times which is a time expensive process when working in the field.

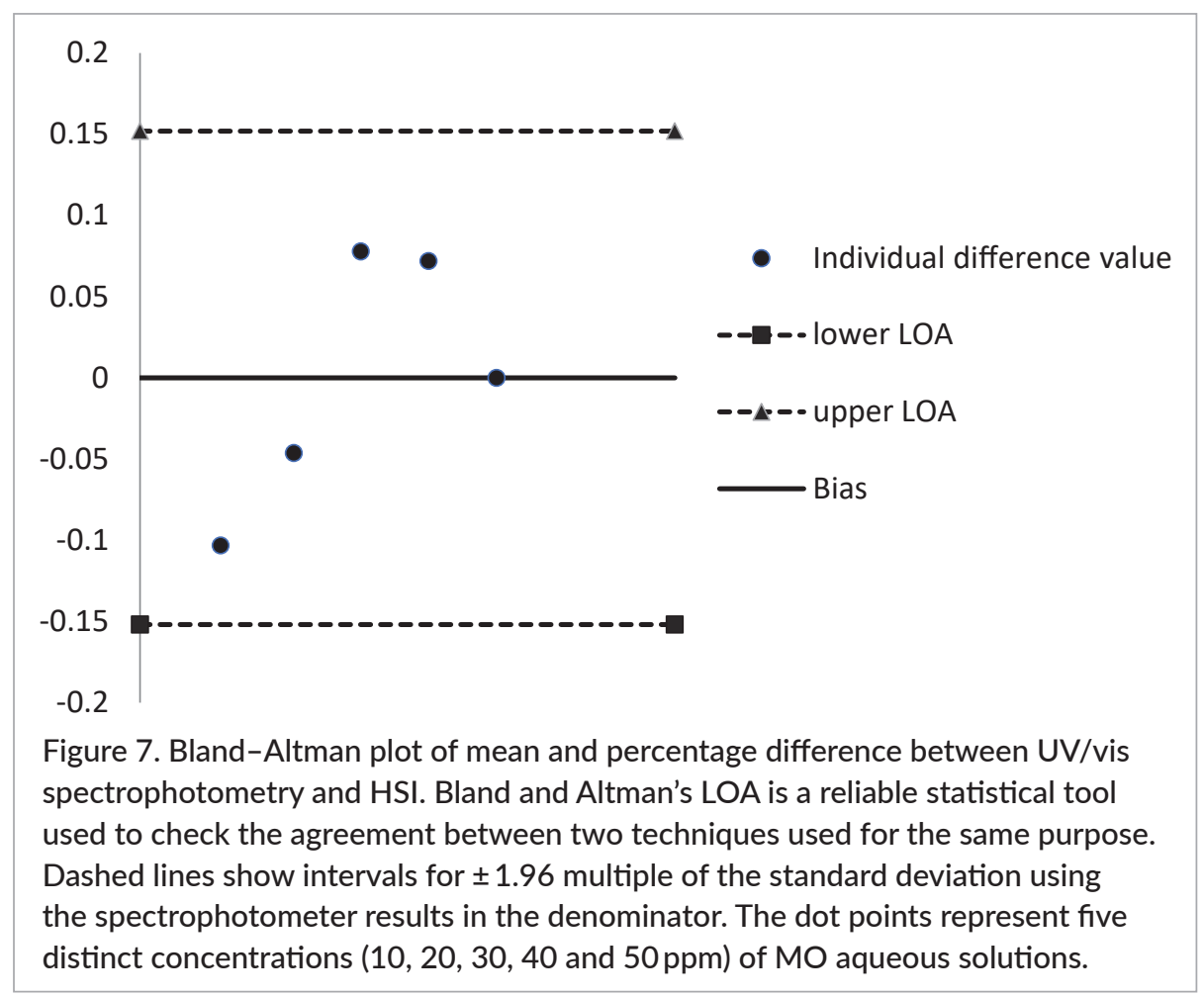


3. The UV-vis device is a laboratory-friendly instrument, yet it is hard to tailor it for the harsh environment of contaminated water produced by industry.

4. HSI overcomes the limit of small quantity of samples by analysing an adjustable field of view for the object of interest. Thus, it saves the processing time for analysis done by the UV/vis spectrophotometer in the laboratory. Therefore, HSI is more appropriate for on-site analysis of industrial contaminated water.

5. More investigation is required to generalise this technique of analysis for inorganic contaminates such as chromates and heavy metals.

\section{Conflict of interest}

Authors have no conflict of interest relevant to this article.

\section{References}

1. E.E. Ebrahiem, M.N. Al-Maghrabi and A.R. Mobarki, "Removal of organic pollutants from industrial wastewater by applying photo-Fenton oxidation technology", Arab. J. Chem. 10, S1674-S1679 (2017). https:// doi.org/10.1016/j.arabjc.2013.06.012

2. Q. He and H. Chen, "Flow injection spectrophotometric determination of anionic surfactants using methyl orange as chromogenic reagent", Fresenius J. Anal. Chem. 367, 270-274 (2000). https://doi. org/10.1007/s002160000330

3. J. Ma, F. Yu, L. Zhou, L. Jin, M. Yang, J. Luan, Y. Tang, H. Fan, Z. Yuan and J. Chen, "Enhanced adsorptive removal of methyl orange and methylene blue from aqueous solution by alkali-activated multiwalled carbon nanotubes", ACS Appl. Mater. Interfaces 4(11), 5749-5760 (2012). https://doi.org/10.1021/ am301053m

4. A.A. Kamaru, N.S. Sani and N.A.N.N. Malek, "Raw and surfactant-modified pineapple leaf as adsorbent for removal of methylene blue and methyl orange from aqueous solution", Desalin. Water Treat. 57(40), 18836-18850 (2015). https://doi.org/10.1080/1944 3994.2015.1095122

5. K.A. Tan, N. Morad and J.Q. Ooi, "Phytoremediation of methylene blue and methyl orange using Eichhornia crassipes", Int. J. Environ. Sci. Dev.
7(10), 724-728 (2016). https://doi.org/10.18178/ ijesd.2016.7.10.869

6. A.S. Alzaydien, "Adsorption behavior of methyl orange onto wheat bran: Role of surface and $\mathrm{pH}$ ", Orient. J. Chem. 31(2), 643-651 (2015). https://doi. org/10.13005/ojc/310205

7. L.V. Trandafilović, D.J. Jovanović, X. Zhang, S. Ptasińska and M.D. Dramićanin, "Enhanced photocatalytic degradation of methylene blue and methyl orange by ZnO:Eu nanoparticles", Appl. Catal. B Environ. 203, 740-752 (2017). https://doi. org/10.1016/j.apcatb.2016.10.063

8. O.S. Bayomie, H. Kandeel, T. Shoeib, H. Yang, N. Youssef and M.M.H. El-Sayed, "Novel approach for effective removal of methylene blue dye from water using fava bean peel waste", Sci. Rep. 10(1), 1-10 (2020). https://doi.org/10.1038/s41598-02064727-5

9. E. Morgounova, B.J. Hackel and D.D. Thomas, "Photoacoustic lifetime contrast between methylene blue monomers and self-quenched dimers as a model for dual-labeled activatable probes monomers and self-quenched dimers as a model for dual-labeled activatable probes", J. Biomed. Opt. 18(5), 056004 (2013). https://doi.org/10.1117/1. JBO.18.5.056004

10. D. Heger and P. Kla, "Aggregation of methylene blue in frozen aqueous solutions studied by absorption spectroscopy", J. Phys. Chem. A 109, 6702-6709 (2005). https://doi.org/10.1021/jp050439j

11. L. Zhai, Z. Bai, Y. Zhu, B. Wang and W. Luo, "Fabrication of chitosan microspheres for efficient adsorption of methyl orange", Chinese J. Chem. Eng. 26(October), 657-666 (2018). https://doi. org/10.1016/j.cjche.2017.08.015

12. S. Naidoo and A.O. Olaniran, "Treated wastewater effluent as a source of microbial pollution of surface water resources", Int. J. Environ. Res. Public Health 11(1), 249-270 (2013). https://doi.org/10.3390/ ijerph110100249

13. A. Yusefi and B. Hatamluyi, "Extraction and determination of methylene blue and methyl orange in wastewaters and food samples by green synthesized nano-iron adsorbent using UV-VIS spectrophotometry", in The 23rd Iranian Seminar of Analytical Chemistry (2016).

14. M.L. Cancillo, A. Serrano, M. Antón, J.A. García, J.M. Vilaplana and B. de la Morena, "An improved 
outdoor calibration procedure for broadband ultraviolet radiometers", Photochem. Photobiol. 81(4), 860 (2005). https://doi.org/10.1562/2005-01-12-RA412R.1

15. E. Dekemper, D. Fussen, B. Van Opstal, J. Vanhamel, D. Pieroux, F. Vanhellemont, N. Mateshvili, G. Franssens, V. Voloshinov, C. Janssen and $\mathrm{H}$, Elandaloussi, "ALTIUS: a spaceborne AOTF-based UV-VIS-NIR hyperspectral imager for atmospheric remote sensing", Proc. SPIE 9241, 92410L (2014). https://doi.org/10.1117/12.2063937

16. R. Abdlaty, J. Orepoulos, P. Sinclair, R. Berman and Q. Fang, "High throughput AOTF hyperspectral imager for randomly polarized light", Photonics 5(1), 3 (2018). https://doi.org/10.3390/photonics5010003

17. R. Abdlaty and Q. Fang, "Acousto-optic tunable filter-based hyperspectral imaging system characterization", Proc. SPIE 10870, 108700Y (2019). https:// doi.org/10.1117/12.2506318

18. R. Abdlaty, S. Sahli, J. Hayward and Q. Fang, "Hyperspectral imaging: Comparison of acousto-optic and liquid crystal tunable filters", Proc. SPIE 10573, 105732P (2018). https://doi. org/10.1117/12.2282532

19. A. Madooei, R.M. Abdlaty, L. DoerwaldMunoz, J. Hayward, M.S. Drew, Q. Fang and J. Zerubia, "Hyperspectral image processing for detection and grading of skin erythema", Proc. SPIE 10133, 1013322 (2017). https://doi. org/10.1117/12.2254132

20. L.M. Dale, E. Andr, C. Boudry, I. Rotar, P. Dardenne, V. Baeten and J.A. Fernández Pierna, "Hyperspectral imaging applications in agriculture and agro-food product quality and safety control", Appl. Spectrosc. Rev. 48, 142-159 (2013). https://doi.org/10.1080/05 704928.2012 .705800

21. P. Mishra, M.S.M. Asaari, A. Herrero-Langreo, S. Lohumi, B. Diezma and P. Scheunders, "Close range hyperspectral imaging of plants: A review", Biosyst. Eng. 164, 49-67 (2017). https://doi.org/10.1016/j. biosystemseng.2017.09.009

22. A. Holmer, C. Homberger, T. Wild and F. Siemers, "Hyperspectral imaging of the degradation of meat and comparison with necrotic tissue in human wounds", J. Spectral Imaging 8, a9 (2019). https://doi. org/10.1255/jsi.2019.a9

23. H. Huang, L. Liu and M.O. Ngadi, "Recent developments in hyperspectral imaging for assessment of food quality and safety", Sensors 14(4), 7248-7276 (2014). https://doi.org/10.3390/s140407248

24. L. Trefan, C. Harris, S. Evans, D. Nuttall, S. Maguire and A.M. Kemp, "A comparison of four different imaging modalities - Conventional, cross polarized, infra-red and ultra-violet in the assessment of childhood bruising", J. Forensic Leg. Med. 59(April), 30-35 (2018). https://doi.org/10.1016/j.jflm.2018.07.015

25. G. Lu and B. Fei, "Medical hyperspectral imaging: a review", J. Biomed Opt. 19(1), 10901 (2014). https:// doi.org/10.1117/1.JBO.19.1.010901

26. M.A. Calin, T. Coman, S.V. Parasca, N. Bercaru, R. Savastru and D. Manea, "Hyperspectral imagingbased wound analysis using mixture-tuned matched filtering classification method", J. Biomed. Opt. 20(4), 046004 (2015). https://doi.org/10.1117/1. JBO.20.4.046004

27. S. Ortega, H. Fabelo, D.K. lakovidis and A. Koulaouzidis, "Use of hyperspectral/multispectral imaging in gastroenterology. Shedding some - different - light into the dark", J. Clin. Med. 8(36), 21 (2019). https://doi.org/10.3390/jcm8010036

28. I. Kopriva, G. Aralica, M. Popović Hadžija, M. Hadžija, L.-I. Dion-Bertrand and X. Chen, "Hyperspectral imaging for intraoperative diagnosis of colon cancer metastasis in a liver", Proc. SPIE 10956, 109560 S (2019). https://doi.org/10.1117/12.2503907 